CERN-TH-7040/93

hepth@xxx/9310095

October 1993

\title{
Exact Spectrum of $S U(n)$ Spin Chain with Inverse-Square Exchange
}

\author{
Alexios P. Polychronakos ${ }^{\dagger}$ \\ Theory Division, CERN \\ CH-1211, Geneva 23, Switzerland
}

\begin{abstract}
The spectrum and partition function of a model consisting of $S U(n)$ spins positioned at the equilibrium positions of a classical Calogero model and interacting through inverse-square exchange are derived. The energy levels are equidistant and have a high degree of degeneracy, with several $S U(n)$ multiplets belonging to the same energy eigenspace. The partition function takes the form of a $q-$ deformed polynomial. This leads to a description of the system in terms of an effective parafermionic hamiltonian, and to a classification of the states in terms of "modules" consisting of base $n$ strings of integers.
\end{abstract}

CERN-TH-7040/93

October 1993

† poly@dxcern.cern.ch 


\section{Introduction}

Systems with interactions of the inverse-square type seem to enjoy a revived popularity. The archetype of such systems is the family of the Calogero-SutherlandMoser integrable systems of particles in one dimension [1-3]. (For an extensive

review and a comprehensive list of earlier references see [4].) A discrete variant of such systems are spin chain models with inverse-square exchange. The original such system is the Haldane-Shastry model [5,6], consisting of $S U(2)$ spins on an equispaced lattice on the circle, interacting through two-body exchange terms inversely proportional to the square of the chord distance. Algebraic aspects of this model have been studied $[7,8]$, and its integrability was shown [9]. Recently, a new model of this type was introduced [10] in which the lattice points lie at the equilibrium positions of classical Calogero particles on a line. Some partial and numerical results on this system were presented in [11]. Furthermore, composite models of this type have appeared [12-15], consisting of generalizations of the Calogero or Sutherland models for particles with spin.

One of the main points of [10] was the observation that the Haldane-Shastry model can be thought of as the high-interaction limit of the Sutherland system with internal degrees of freedom. The spatial degrees of freedom in this limit decouple and the remaining spin degrees of freedom constitute the desired spin chain lattice. This fact was exploited in [16] in order to solve the Haldane-Shastry model in the thermodynamic limit (number of sites $\rightarrow \infty$ ). In this paper, we will use it to fully solve the spin chain model proposed in [10]. 


\section{Review of the Calogero system of particles with $S U(n)$ spins}

We briefly review here the Calogero-type system of particles with spin and its spectrum, which was first introduced and solved in [14]. Consider $N$ particles on the line with internal degrees of freedom ("spin") transforming under the fundamental of $S U(n)$. The hamiltonian of the system is

$$
H=\frac{1}{2} \sum_{i}\left(p_{i}^{2}+\omega^{2} x_{i}^{2}\right)+\sum_{i<j} \frac{l\left(l-M_{i j}\right)}{\left(x_{i}-x_{j}\right)^{2}}
$$

where $M_{i j}$ is the operator that exchanges the positions of particles $i$ and $j$. Define also the operators $\sigma_{i j}$ which exchange the spins of particles $i$ and $j$. In terms of the fundamental $S U(n)$ generators for each particle, $\sigma_{i}^{a}\left(a=1,2, \ldots n^{2}-1\right)$, the $\sigma_{i j}$ have the expression

$$
\sigma_{i j}=\frac{1}{n}+\sum_{a} \sigma_{i}^{a} \sigma_{j}^{a}
$$

For the above hamiltonian, there are raising and lowering operators $a_{i}^{\dagger}$ and $a_{i}$, defined

$$
a_{i}^{\dagger}=p_{i}+\sum_{j \neq i} \frac{i}{x_{i}-x_{j}} M_{i j}+i \omega x_{i}, \quad a_{i}=p_{i}+\sum_{j \neq i} \frac{i}{x_{i}-x_{j}} M_{i j}-i \omega x_{i},
$$

satisfying

$$
\begin{gathered}
{\left[a_{i}, a_{j}\right]=\left[a_{i}^{\dagger}=a_{j}^{\dagger}\right]=0, \quad\left[a_{i}, a_{j}^{\dagger}\right]=2 \omega \delta_{i j}\left(1+l \sum_{k \neq j} M_{i k}\right)-2 \omega\left(1-\delta_{i j}\right) M_{i j},} \\
{\left[H, a_{i}\right]=-\omega a_{i}, \quad\left[H, a_{i}^{\dagger}\right]=\omega a_{i}^{\dagger} .}
\end{gathered}
$$

Therefore, the energy eigenstates of $H$ can in principle be found in a systematic way once the ground state is determined. Since $H$ is invariant under total particle permutation, we can choose the states to be bosonic. On such states the 
total exchange operator $M_{i j} \sigma_{i j}$ becomes one, and thus $M_{i j}=\sigma_{i j}$ and the above hamiltonian becomes

$$
H=\frac{1}{2} \sum_{i}\left(p_{i}^{2}+\omega x_{i}^{2}\right)+\sum_{i<j} \frac{l\left(l-\sigma_{i j}\right)}{\left(x_{i}-x_{j}\right)^{2}} .
$$

This hamiltonian commutes with the total spin $S^{a}=\sum_{i} \sigma_{i}^{a}$. We shall call the case $l>0$ ferromagnetic and the one $l<0$ antiferromagnetic.

(Note that in [14] the choice of fermionic states was made. Due to the singular nature of the interaction in (2.6), there is no particle penetration and thus the results will not depend on this choice; the penetrability of the original hamiltonian (2.1), which was due to the exchange operators $M_{i j}$, has been traded for a flow between different spin sectors.)

In the ferromagnetic regime, the ground state is annihilated by all $a_{i}$ and acquires the form

$$
\psi_{f}=\prod_{i<j}\left|x_{i}-x_{j}\right|^{l} \exp \left(-\frac{1}{2} \sum_{i} \omega x_{i}^{2}\right) \chi(S)
$$

where $\chi$ is any component of the fully symmetric representation in the tensor product of fundamentals $\sigma_{1} \sigma_{2} \cdots \sigma_{N}$, that is, the $N$-fold symmetric irrep of $S$. Thus, the ferromagnetic ground state $\psi_{f}$ is $\frac{(n+N-1) !}{(n-1) ! N !}-$ fold degenerate. Excited states can be obtained by acting on the ground states with symmetric combinations of the raising operators, that is, polynomials of the operators

$$
A_{k} \equiv \sum_{i}\left(a_{i}^{\dagger}\right)^{k} \quad \text { and } \quad A_{k}^{a} \equiv \sum_{i} s_{i}^{a}\left(a_{i}^{\dagger}\right)^{k}
$$

which satisfy the commutation relations

$$
\left[A_{k}, A_{r}\right]=\left[A_{k}, A_{r}^{a}\right]=0, \quad\left[A_{k}^{a}, A_{r}^{b}\right]=f^{a b c} A_{k+r}^{c}
$$

with $f^{a b c}$ the structure constants of $S U(n)$. Both $A_{k}$ and $A_{k}^{a}$ create $k$ quanta of energy, but $A_{k}^{a}$ also alters the $S U(n)$ representation by moving one box in its Young tableau. For an explicit treatment of the $S U(2)$ case, see [14]. 
The problem of determining the energy eigenstates can be substantially simplified by the following observation: the above procedure is, in fact, identical to the one in the free particle case (without couplings). Indeed, the ground state (2.7) is in the same representation as the free one (take $l=0$ ), and the free excited states can be found by acting with the same creation operators $A_{k}$ and $A_{k}^{a}$ (note that the commutation relations (2.9) of these operators are independent of $l$ ). In the free case, the internal degrees of freedom simply become dynamically irrelevant particle "flavors." Thus, the states of the system can be found by considering $N$ free bosons in an external harmonic oscillator potential, each one being in one of $n$ possible flavors. If $N_{k}$ such bosons are in the same level of the harmonic oscillator spectrum, they obviously transform under the $N_{k}$-fold symmetric irrep of $S U(n)$. The representation of the full state then is the tensor product of the symmetric representations for each filled level. (In the ground state all particles are lumped together in the lowest level, thus $N_{k}=N$ and we get the irrep of (2.7).) The only difference with the free system is an overall shift of the spectrum $E_{o}$, accounting for the different ground state energy.

In the case $l<0$ the above ground state (2.7) is unacceptable due to the negative power in the exponent of $\left|x_{i}-x_{j}\right|$. Notice, however, that the antiferromagnetic hamiltonian (2.6) can be achieved by turning $l$ into $-l>0$ and choosing the statistics of the particles to be fermionic $\left(M_{i j} \sigma_{i j}=-1\right)$. Therefore, generalizing the result of [14] for the $S U(2)$ case (see also [12]), the antiferromagnetic ground state is

$$
\psi_{a f}=\prod_{i<j}\left|x_{i}-x_{j}\right|^{-l} \psi_{F}\left(\left\{x_{i}, s_{i}^{a}\right\}\right),
$$

where $\psi_{F}$ is the ground state of a system of $N$ free fermions with $n$ flavors in an external harmonic oscillator potential. This state is in the $m$-fold antisymmetric representation of $S U(n)$, where $m=N \bmod n$. The excited states can be found by acting with the same totally symmetric operators as in the ferromagnetic case. Again, we can explicitly find these states by considering the states of the corresponding free fermion problem and shifting all energy levels by the corresponding 
ground state energy. In each oscillator level we can put $N_{k}$ fermions, where $N_{k}$ is at most $n$. Such a multiplet transforms now under the $N_{k}$-fold antisymmetric irrep of $S U(n)$, and the total state carries the tensor product of irreps for all occupied oscillator levels. The ground state is $\frac{n !}{(n-m) ! m !}$-fold degenerate.

An interesting observation is that the states (2.7) and (2.10) are actually valid

only for $|l|>\frac{1}{2}$ (this is related to the "spurious" states in [14]). The reason is that hermiticity of the hamiltonian requires the particle current to vanish at coincidence points $x_{i}=x_{j}$, and this will happen if the power of $\left|x_{i}-x_{j}\right|$ is greater than $\frac{1}{2}$. In fact, since the eigenstates of the ferromagnetic and antiferromagnetic cases are quite different, an intricate level-crossing must take place in the region $|l|<\frac{1}{2}$ in order to match the two. This is irrelevant in our case, since we will be interested in the limit $l \rightarrow \pm \infty$. It remains, however, a very interesting problem to determine the spectral flow of this theory in the interval $\left[-\frac{1}{2}, \frac{1}{2}\right]$.

\section{The spin chain system}

We come now to our main object. As was pointed out in [10], in the strong coupling constant limit the coordinate degrees of freedom of this system decouple from the spin ones; this is because, for very large $l$, the repulsion between particles becomes dominant and, unless a very high number of quanta are excited, the coordinates of the particles assume their classical equilibrium value. Thus, in this limit the coefficients of the spin couplings become nondynamical constants and the spin degrees of freedom decouple. Therefore, the above system becomes the tensor product of a spinless Calogero system and of a spin chain system, with spins lying at the equilibrium positions of the classical Calogero system. Note that $\omega$ should also be scaled by a factor of $|l|$ in order to have a nontrivial limit. Dropping an overall scaling factor of $|l|$, then, the hamiltonian of the spin chain system is

$$
H_{s}=-\operatorname{sgn}(l) \sum_{i<j} \frac{\sigma_{i j}}{\left(x_{i}-x_{j}\right)^{2}}
$$


where the $x_{i}$ minimize the Calogero potential, that is,

$$
\omega x_{i}-\sum_{i<j} \frac{2}{\left(x_{i}-x_{j}\right)^{3}}=0 .
$$

The energy levels of the full system can be expressed as

$$
E_{s, p}=E_{s}+E_{p}
$$

where $E_{s}$ are the energy levels of the spin chain system and $E_{p}$ are the levels of the Calogero system. Obviously, the states corresponding to $E_{p}$ carry no spin, and therefore the full spin content of the level $E_{s}$ coincides with the one of $E_{s, p}$. Since both $E_{s, p}$ and $E_{p}$ have an equidistant spectrum, it already follows that the spectrum of the spin chain $E_{s}$ is also equidistant.

The nice feature of the spectrum of the full system is that it is essentially the same for all values $|l|>\frac{1}{2}$, namely the spectrum of a free system, depending nontrivially only on the sign of $l$. Indeed, the spacing of the levels contains an overall factor $|l|$ (due to $\omega \rightarrow|l| \omega$ ), which we will drop as we did in (3.1). We will also take $\omega=1$ from now on. There is also an $l$-dependent overall shift of the spectrum, due to the ground state energy, which is irrelevant for the factorization (3.3). Thus, the factorization (3.3) holds even for finite values of $l$ (of course, the wavefunctions in general will only factorize in the $|l| \rightarrow \infty$ limit). Therefore, we reached the conclusion that the energy eigenstates of the above spin chain system can be found by taking the states of a free $N$-particle system with $n$ flavors and "modding" them by the states of a corresponding system with one flavor. Taking the particles to be bosons (fermions) allows us to examine the spin system from the ferromagnetic (antiferromagnetic) point of view, respectively. In what follows we will implement this procedure to explicitly find the spin system's spectrum and partition function.

Let $Z_{n, N}^{B}\left(Z_{n, N}^{F}\right)$ be the partition function of the bosonic (fermionic) $N$-particle $n$-flavor problem for inverse temperature $\beta$, and $Z_{n, N}^{f}\left(Z_{n, N}^{a f}\right)$ the partition function 
of the spin chain ferromagnetic (antiferromagnetic) system. Then, (3.3) implies that

$$
Z_{n, N}^{B}=Z_{n, N}^{f} Z_{1, N}^{B}
$$

and similarly for the $F$, af case. For the bosonic case, dropping the zero-point energy of the harmonic oscillator, the $N$-boson partition function is given by

$$
Z_{1, N}^{B}=\sum_{k_{i} \leq k_{i+1}} \prod_{i=1}^{N} q^{k_{i}}
$$

where $q=e^{-\beta}$. By changing variables to $p_{i}=k_{i}-k_{i-1}, p_{1}=k_{1}$, we obtain

$$
Z_{1, N}^{B}=\sum_{p_{i}} \prod_{i=1}^{N} q^{(N-i+1) p_{i}}=\prod_{k=1}^{N} \frac{1}{1-q^{k}}
$$

To find $Z_{n, N}^{B}$, we remark that the grand canonical partition function of the $n$-flavor bosonic system is the product of $n$ one-flavor ones, thus

$$
\mathcal{Z}_{n}^{B}=\sum_{N=0}^{\infty} Z_{n, N}^{B} y^{N}=\left(\mathcal{Z}_{1}^{B}\right)^{n}=\left(\sum_{N=0}^{\infty} Z_{1, N}^{B} y^{N}\right)^{n}
$$

where $y=e^{-\mu}$ with $\mu$ the chemical potential. Therefore

$$
Z_{n, N}^{B}=\sum_{\sum_{i} k_{i}=N} \prod_{i=1}^{n} Z_{1, k_{i}}^{B}
$$

Combining (3.4), (3.6) and (3.8) we finally obtain

$$
Z_{n, N}^{f}=\sum_{\sum_{i} k_{i}=N} \frac{\prod_{k=1}^{N}\left(1-q^{k}\right)}{\prod_{i=1}^{n} \prod_{r=1}^{k_{i}}\left(1-q^{r}\right)}
$$

For the fermionic case, the single-flavor partition function is identical to the bosonic one (this is a particular case of bosonization in one dimension, since the 
oscillator spectrum is "relativistic"), the only difference being the ground state energy. Therefore,

$$
Z_{1, N}^{F}=q^{\frac{N(N-1)}{2}} \prod_{k=1}^{N} \frac{1}{1-q^{k}}
$$

The rest of the argument is the same as in the bosonic case. (Note that the inclusion of the ground state energy in (3.10) is necessary to get the correct $n$-flavor partition function in the fermionic version of (3.8).) We obtain

$$
Z_{n, N}^{a f}=q^{-E_{n, N}^{a f}} \sum_{\sum_{i} k_{i}=N} \prod_{k=1}^{N}\left(1-q^{k}\right) \prod_{i=1}^{n} \frac{q^{\frac{1}{2}\left(k_{i}-\frac{N}{n}\right)^{2}}}{\prod_{r=1}^{k_{i}}\left(1-q^{r}\right)} .
$$

In the above, we had to subtract explicitly the zero-point energy of the partition function, so as to bring the ground state to zero energy. This was necessary since the fermionic systems used to derive (3.11) had a nonzero ground state energy. The constant $E_{n, N}^{a f}$ is calculated to be

$$
E_{n, N}^{a f}=\frac{m(n-m)}{2 n}, \quad m=N \bmod n .
$$

A number of nontrivial checks can be performed on the above partition functions. Firstly, since they are partition functions of a finite system with equidistant levels, they should be a polynomial in $q$. This may not be obvious from the expressions (3.9) and (3.11), but it will be proved in a short while. Next, the value of either $Z_{n, N}^{f}$ or $Z_{n, N}^{a f}$ for $q=1$ should reproduce the number of states of the system. Indeed, in that limit, (3.9) and (3.11) simply become the polynomial expansion of

$$
n^{N}=(1+1+\cdots 1)^{N}=\sum_{\sum_{i} k_{i}=N} \frac{N !}{\prod_{i=1}^{n} k_{i} !} .
$$

Finally, the two partition functions really describe the same problem, with the 
spectrum reversed. This means that

$$
Z_{n, N}^{a f}(q)=q^{E_{\max }} Z_{n, N}^{f}\left(q^{-1}\right)
$$

where $E_{\max }$ is the energy of the highest excited state in either case. By isolating the highest power of $q$ in $(3.9)$, we find

$$
E_{\max }=\frac{n-1}{2 n} N^{2}-\frac{m(n-m)}{2 n} .
$$

It is easy to check that (3.14) indeed holds for the expressions (3.9) and (3.11).

\section{The $S U(2)$ case}

For the special case of $S U(2)$, the partition functions take the form

$$
\begin{gathered}
Z_{2, N}^{f}=\sum_{k=0}^{N} \prod_{r=1}^{k} \frac{1-q^{N-r+1}}{1-q^{r}} \\
Z_{2, N}^{a f}=q^{-\frac{m}{4}} \sum_{k=0}^{N} q^{\left(k-\frac{N}{2}\right)^{2}} \prod_{r=1}^{k} \frac{1-q^{N-r+1}}{1-q^{r}} .
\end{gathered}
$$

Formula (4.2) reproduces the partition function proposed in [11] on the basis of numerical investigations (modulo a ground-state energy correction for odd $N$ ).

Some properties of the $S U(2)$ spectrum are easy to infer directly from the corresponding particle system. From the ferromagnetic end, it is clear that the first irrep of spin $\frac{N}{2}-k$ will appear at energy $k$, coming from the state where $k$ bosons have been excited to the first excited state, which carries the representation $\left(\frac{N-k}{2}\right) \otimes\left(\frac{k}{2}\right)$ (numbers in parentheses correspond to spin). Similarly, from the antiferromagnetic end, the first irrep of spin $\frac{m}{2}+k$ will appear at energy $k(k+m)$, corresponding to $k$ fermions excited to successive levels above the Fermi level and leaving $k$ "holes" behind, which carries the representation $\left(\frac{1}{2}\right) \otimes \cdots\left(\frac{1}{2}\right)(2 k$ times $)$. 
The creation operator picture is also useful; clearly the set of all polynomials in $A_{k}, A_{k}^{a}$, modded by the set of all polynomials in $A_{k}$ (the single-flavor case), leaves the set of all polynomials in $A_{k}^{a}$. Each such operator, acting on a state of total spin $s$ will produce a state of spin $s-1$ [14], as long as $s \geq 1$. The states of the first $\frac{N}{2}$ energy levels in the ferromagnetic case can be generated this way. Counting all possible monomials of degree $k$, we find that the subspace of the Hilbert space with energy $k$ decomposes as

$$
\mathcal{H}_{k}=\sum_{i=1}^{k} \oplus\left(\frac{N}{2}-i\right)_{p(k, i)}
$$

where $p(k, i)$ denotes the number of partitions of $k$ into $i$ positive integers and counts the multiplicity of each spin.

We emphasize here that the partition functions (4.1) and (4.2) reproduce the full representation content of the energy levels. To see this, remember that the sum over $k$ in the above partition functions came from the sum over $k$ in (3.8) (for $N=2$ ). Therefore, each term represents a state with $k$ particles of the one flavor and $N-k$ particles of the other. Since "flavors" correspond to the different spin states of the particles, each term $k$ corresponds to states with total 3-component of spin $S^{3}=k \frac{1}{2}+(N-k)\left(-\frac{1}{2}\right)=k-\frac{N}{2}$. Thus, the above sum groups the energy levels in terms of the 3 -component of their total spin $S^{3}$. Once we have all the states in each energy level and their spins, there is always a unique way to group them into representations of the total spin.

Having realized that, it is trivial to write the partition function of the system in the presence of a magnetic field $B$ in the 3 -direction, as

$$
Z_{n, N}^{f}(q, w)=\sum_{k=0}^{N} w^{\left(k-\frac{N}{2}\right)} \prod_{r=1}^{k} \frac{1-q^{N-r+1}}{1-q^{r}}, \quad w=e^{-\beta B}=q^{B}
$$

and similarly for the antiferromagnetic case. An interesting property of the system stemming from the above is the following: if a "global" interaction between 
the spins is included, of the form $-\left(S^{3}\right)^{2}$, then the antiferromagnetic system is transformed into the ferromagnetic one. This is very peculiar: the presence of such an interaction breaks the global $S U(2)$ invariance. Therefore we do not expect the states to fall into $S U(2)$ multiplets, while they obviously do in this particular case since the spectrum is identical to the $S U(2)$-invariant ferromagnet. Obviously the multiplets obtained this way will not be representations of the global $S U(2)$, but there should be some nontrivially-defined set of $S U(2)$ generators in this case which commute with the hamiltonian.

A useful observation about the partition function (4.4) is that has the form of a $q$-binomial. We remind the reader that the $q$-deformation of a number is, in one definition [17],

$$
[x]_{q}=\frac{q^{x}-1}{q-1}
$$

and the $q$-factorial

$$
[k !]_{q}=\prod_{r=1}^{k}[r]_{q} .
$$

From this, we define the $q$ - $(m$-choose- $n)$ symbol

$$
\left[\begin{array}{l}
m \\
n
\end{array}\right]_{q}=\frac{[m !]_{q}}{[n !]_{q}[(m-n) !]_{q}}
$$

and the $q$-binomial as

$$
[1+w]_{q}^{N}=\sum_{k=1}^{N}\left[\begin{array}{l}
N \\
k
\end{array}\right]_{q} w^{k}
$$

It is easy to see that, up to an overall factor $w^{-\frac{N}{2}}$, the ferromagnetic $Z_{2, N}^{f}(q, w)$ is exactly the $q$-binomial of the $N$-th degree for $w$. This is useful in finding a factorized form for $Z_{2, N}^{f}$. Indeed, for an alternative symmetric definition of the 
$q$-deformation

$$
[[x]]_{q}=\frac{q^{\frac{x}{2}}-q^{-\frac{x}{2}}}{q^{\frac{1}{2}}-q^{-\frac{1}{2}}}=q^{\frac{1-x}{2}}[x]_{q},
$$

it is known that the corresponding $q$-binomial assumes the form

$$
\left[\left[1+q^{\frac{N-1}{2}} w\right]\right]_{q}^{N}=\prod_{k=0}^{N-1}\left(1+q^{k} w\right)
$$

The above formula admits the interpretation of the grand canonical partition function of a fermionic system with levels $E=0,1, \ldots N-1$ and chemical potential $e^{-\mu}=w$. Using (4.9), on the other hand, we can see that the ferromagnetic partition function differs from the above $q$-binomial by an extra factor $q^{\frac{k(k-1)}{2}}$ in each term. From (4.10), then, we are led to an effective description of the system as a fermionic system with $N$ levels and hamiltonian

$$
H_{e f f}=\sum_{k=0}^{N}(k+B) n_{k}-\sum_{k<l} n_{k} n_{l}
$$

where the occupation numbers $n_{k}$ take the values 0,1 . The corresponding antiferromagnetic effective hamiltonian can be found by taking $H_{\text {eff }} \rightarrow-H_{\text {eff }}$ and adding $E_{\max }=\frac{N^{2}}{4}-\frac{m}{4}$. This reproduces, for $B=0$, the model derived in [11] on the basis of numerical results. Other useful properties of the partition function can be derived by using $q$-function results. For instance, by acting with a $q$-derivative on $Z_{2, N}^{f}(B)$ we find the recursion relation

$$
Z_{2, N}^{f}(B+1)=Z_{2, N}^{f}(B)-[N]_{q} q^{\frac{B}{2}}(1-q) Z_{2, N-1}^{f}(B)
$$

A corresponding relation for the antiferromagnetic case is easily obtained. 


\section{The $S U(n)$ case}

The general $S U(n)$ case can be examined in an analogous way. Firstly, the partition function (3.9) (or (3.11)) also fully determines the $S U(n)$ representation content of the spectrum. The "flavor" of the particles in $Z_{n, N}^{B}$ corresponds to the $n$ different components of the $S U(n)$ spin of each particle, and therefore the set

of $n-1$ independent quantities $k_{i}-\frac{N}{n}$ determines the values of the $n-1$ Cartan generators of $S U(n)$, in the nonorthogonal fundamental parametrization

$$
\left(H^{i}\right)_{j k}=\delta_{i j} \delta_{i k}-\frac{1}{n}
$$

(the above are just the $U(n)$ Cartan generators minus $\frac{Q}{n}$, with $Q$ the $U(1)$ charge). These are enough to fully determine the $S U(n)$ state. Once all these states are known for each energy level, they can be assembled into irreps of $S U(n)$. We can also introduce an $S U(n)$ magnetic field interaction in the problem, as

$$
H_{\text {mag }}=\sum_{a=1}^{N^{2}-1} S^{a} B^{a} .
$$

With a global $S U(n)$ transformation we can always rotate $B^{a}$ in the Cartan subspace, and the interaction takes the symmetric form

$$
H_{\text {mag }}=\sum_{i=1}^{N} k_{i} B_{i} \quad \text { with } \quad \sum_{i} B_{i}=0
$$

So the full partition function takes the form

$$
Z_{n, N}^{f}\left(q,\left\{w_{i}\right\}\right)=\sum_{\sum_{i} k_{i}=N}\left[\begin{array}{c}
N \\
\left\{k_{i}\right\}
\end{array}\right] \prod_{q}^{n} w_{i}^{k_{i}}
$$

where $w_{i}=e^{-\beta B_{i}}$ and we defined the generalized $q$-choose symbol

$$
\left[\begin{array}{c}
N \\
\left\{k_{i}\right\}
\end{array}\right]_{q}=\frac{[N !]_{q}}{\prod_{i}\left[k_{i} !\right]_{q}} .
$$


Similarly, for the antiferromagnetic case we obtain

$$
Z_{n, N}^{a f}\left(q,\left\{w_{i}\right\}\right)=q^{-E_{n, N}^{a f}} \sum_{\sum_{i} k_{i}=N}\left[\begin{array}{c}
N \\
\left\{k_{i}\right\}
\end{array}\right] \prod_{i=1}^{n} q^{\left(k_{i}-\frac{N}{n}\right)^{2}} w_{i}^{k_{i}} .
$$

Again we observe that an extra interaction of the form $\sum_{i}\left(H^{i}\right)^{2}$ turns the system from ferromagnetic to antiferromagnetic.

From the above, it follows that the $S U(n)$ partition function is the $q$-polynomial of the $N$-th degree in the variables $w_{i}$. A systematic way of writing this polynomial, other than (5.4), is the following: consider the variables $X_{i}, i=1, \cdots n$, obeying the $q$-commutation relations

$$
X_{i} X_{j}=q X_{j} X_{i} \quad \text { for } \quad i>j .
$$

Then the ordinary polynomial expansion of $\left(X_{1}+\cdots X_{n}\right)^{N}$ automatically reproduces the $q$-polynomial, once the variables in each monomial are ordered in ascending index order. This proves, in particular, that the partition function is a polynomial in $q$ since the above will manifestly produce polynomial coefficients.

The previous construction allows us to find a model hamiltonian for this system. Consider $N$ levels, all at zero energy, ordered from 1 to $N$. In each level place exactly one particle of flavor $i=1, \cdots n$, and for every "reversal of order" in the configuration increase the energy by one. That is, for each pair of particles such that the one of a lower flavor is in a higher level than the other, the energy increases by one. The model hamiltonian in terms of occupancies $n_{i, k}, i$ labeling the flavor and $k$ the level, takes the form

$$
H_{e f f}^{f}=\sum_{i, k} B_{i} n_{i, k}+\sum_{k<l, i>j} n_{i, k} n_{j, l}, \quad n_{i, k}=0,1, \quad \sum_{i} n_{i, k}=1 .
$$

Alternatively, we can describe the system in terms of parafermions of order $n$, the flavor index of the previous model becoming the occupancy. In this language, the 
hamiltonian becomes

$$
H_{e f f}=\sum_{k} B_{1+n_{k}}+\sum_{k<l} \theta\left(n_{k}-n_{l}\right), \quad n_{k}=0,1, \cdots n-1
$$

with the step function $\theta$ defined

$$
\theta(x)=\left\{\begin{array}{l}
1 \text { for } x>0 \\
0 \text { for } x \leq 0
\end{array}\right.
$$

One can see that the case $n=2$ is, up to a constant, equivalent to the model given earlier in (4.11) with $B_{1}=-B_{2}=\frac{B}{2}$. Therefore, the states of the $S U(n)$ problem can be described in terms of $N$-digit numbers in the $n$-basis ("modules") $n_{1} \cdots n_{N}$, which determine the energy as well as the $S U(n)$ quantum numbers of the state. In terms of these modules and the hamiltonian (5.9), it follows that the ground state consists of all totally ordered configurations $0 \ldots 1 \ldots(n-1)$, with an otherwise arbitrary multiplicity of each occupancy. There are obviously $(N+n-1-$ choose- $n-1$ ) such configurations, reproducing the degeneracy of the ferromagnetic ground state. The highest excited state is the one with the "maximal reversal of order," that is, the state $(n-1) \ldots 1 \ldots 0$, with a multiplicity of each occupancy as close to $\frac{N}{n}$ as possible. There are $(n$-choose- $m)$ such modules, and this reproduces the degeneracy of the antiferromagnetic ground state.

\section{Epilogue}

In conclusion, we see that the above system exhibits a simple but very rich structure. Unlike the standard Haldane-Shastry model, its spectrum is equallyspaced and therefore simpler. The highly degenerate structure of the levels, on the other hand ("supermultiplets") is a feature common to the two models, although they differ in the actual representation content of the levels. We also remark that the present system does not have a (lattice) translation invariance. It is therefore remarkable that, in spite of this fact, it is amenable to a complete solution, 
for arbitrary $n$ and $N$. There are still, however, many interesting issues to be addressed. Among them are a systematic, group-theoretic procedure of finding the level structure in the antiferromagnetic regime, its relation to conformal field theory, the statistical mechanics of the $S U(n)$-magnetic field model, and the identification and study of spinon excitations.

Acknowledgements: I am thankful to A.M. Perelomov for reviving my interest in this problem, to C. Zachos for a crash-tutorial on $q$-functions and their properties, and to J. Minahan for a critical reading of the manuscript.

\section{REFERENCES}

1. F. Calogero, J. Math. Phys. 102191 (1969); 102197 (1969); 12419 (1971); Lett. Nuovo Cimento 13, 411 (1975); F. Calogero and C. Marchioro, Lett. Nuovo Cimento 13, 383 (1975).

2. B. Sutherland, Phys. Rev. A 4, 2019 (1971); 5, 1372 (1972); Phys. Rev. Lett. 34, 1083 (1975).

3. J. Moser, Adv. Math. 16, 1 (1975).

4. M.A. Olshanetsky and A.M. Perelomov, Phys. Rep. 71, 314 (1981); 94, 6 (1983).

5. F.D.M. Haldane, Phys. Rev. Lett. 60, 635 (1988); 66, 1529 (1991).

6. B.S. Shastry, Phys. Rev. Lett. 60, 639 (1988); 69, 164 (1992).

7. V.I. Inozemtsev, J. Stat. Phys. 59, 1143 (1989).

8. F.D.M. Haldane, Z.N.C. Ha, J.C. Talstra, D. Bernard and V. Pasquier, Phys. Rev. Lett. 69, 2021 (1992).

9. M. Fowler and J.A. Minahan, Phys. Rev. Lett. 70, 2325 (1993).

10. A.P. Polychronakos, Phys. Rev. Lett. 70, 2329 (1993).

11. H. Frahm, J. Phys. A. 26, 473 (1993). 
12. Z.N.C. Ha and F.D.M. Haldane, Phys. Rev. B 46, 9359 (1992).

13. N. Kawakami, Phys. Rev. B 46, 1005 (1992); 46, 3191 (1992).

14. J.A. Minahan and A. P. Polychronakos, Phys. Lett. B 302, 265 (1993).

15. K. Hikami and M. Wadati, Phys. Lett. A 173, 263 (1993).

16. B. Sutherland and B. S. Shastry, Phys. Rev. Lett. 71, 5 (1993).

17. All the relevant formulae and relations used in this context can be found in H. Exton, "q-Hypergeometric Functions and Applications," Ellis-Horwood/Wiley, 1983. 